\title{
Device Saturation Behavior of Submillimeter-Wave Membrane Photonic Transmitters
}

\author{
Ming-Chun Tien, Hsu-Hao Chang, Ja-Yu Lu, Li-Jin Chen, Shih-Yuan Chen, Ruey-Beei Wu, Senior Member, IEEE, \\ Wei-Sheng Liu, Jen-Inn Chyi, Senior Member, IEEE, and Chi-Kuang Sun, Senior Member, IEEE
}

\begin{abstract}
Ultrahigh-speed photodetectors and printed-circuit antennas construct photonic transmitters. In this letter, we studied the saturation behaviors of an edge-coupled membrane photonic transmitter based on low-temperature-grown GaAs. The saturation behaviors determine the optimized operation condition of photonic transmitters. Ultrahigh external light-terahertz (THz) conversion efficiency of $0.11 \%$ was achieved with $645-\mathrm{GHz}$ radiation. According to our knowledge, this value is the highest reported external conversion efficiency of all photonic transmitters with radiation higher than $500 \mathrm{GHz}$. The high conversion efficiency and the edge-coupled structure of our devices release the power burden imposed on tunable semiconductor laser sources and imply their applications as compact all-solid-state $\mathrm{THz}$ radiation sources.
\end{abstract}

Index Terms-Low-temperature-grown GaAs (LTG-GaAs), millimeter-wave, photonic transmitter, submillimeter-wave, terahertz (THz), traveling wave photodetectors.

$\mathbf{T}$ ERAHERTZ $(\mathrm{THz})$ technology has recently attracted a lot of attention and a compact high-power high-efficiency $\mathrm{THz}$ emitter is important to promote its applications. Compared with other millimeter- or submillimeter-wave emission techniques such as Gunn diodes [1], p-type Ge-based or quantum cascade THz lasers [2], [3], and resonant tunneling diodes [4], photonic transmitters have the advantages of simplicity, roomtemperature operation, tunable $\mathrm{THz}$ wavelength, and integratability with other semiconductor devices to become compact $\mathrm{THz}$ sources. Low-temperature-grown GaAs (LTG-GaAs) has been utilized to fabricate photonic transmitters due to its ability to operate in the $\mathrm{THz}$ frequency regime (corresponding to 100 to $1000-\mu \mathrm{m}$ optical wavelength), which lies beyond the capabilities of both solid-state laser on the short-wavelength side and of electronic sources such as Gunn or IMPATT diodes [1] on the long-wavelength side. Recently, we have demonstrated a novel edge-coupled membrane photonic transmitter based on LTG-GaAs and an external light-THz conversion efficiency of $2 \times 10^{-4}$ at a $1.6-\mathrm{THz}$ radiation frequency was achieved [5]. LTG-GaAs based photonic transmitters also have been applied in $\mathrm{THz}$ image systems and $\mathrm{THz}$ spectrometers [6], [7].

Manuscript received August 4, 2003; revised November 6, 2003. This work was supported by the Institute of Applied Science and Engineering Research, Academic Sinica, Taiwan, R.O.C.

M.-C. Tien, H.-H. Chang, J.-Y. Lu, L.-J. Chen, and C.-K. Sun are with the Department of Electrical Engineering and Graduate Institute of Electro-Optical Engineering, National Taiwan University, Taipei 10617, Taiwan, R.O.C. (e-mail: sun@cc.ee.ntu.edu.tw).

S.-Y. Chen and R.-B. Wu are with the Graduate Institute of Communication Engineering, National Taiwan University, Taipei 10617, Taiwan, R.O.C.

W.-S. Liu and J.-I. Chyi are with the Department of Electrical Engineering, National Central University, Jung-Li 300, Taiwan, R.O.C.

Digital Object Identifier 10.1109/LPT.2004.823774

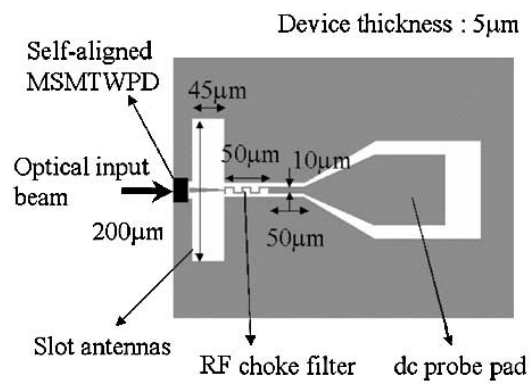

Fig. 1. Structure of the edge-coupled membrane photonic transmitter. Geometric information for the $650-\mathrm{GHz}$ device is provided.

In this letter, we study the conversion efficiency saturation behavior of the edge-coupled membrane photonic transmitters. The study was performed on devices with improved design compared with those previously published in [5] and the new resonant frequency was designed at $645 \mathrm{GHz}$. The external light- $\mathrm{THz}$ conversion efficiency is a function of detector bias, optical excitation power, and antenna design. Under high bias voltage or high optical excitation, the conversion efficiency of the device saturates due to the carrier lifetime increasing effect in LTG-GaAs [8], [9]. The aim of this work is to find the optimum operation conditions of the device, such as applied bias voltage and excitation optical power, to maximize external conversion efficiency.

Fig. 1 shows the structure of the new membrane photonic transmitter, which is composed of a high-speed photodetector, a radio frequency (RF) choke filter, and a planar antenna. We adopted metal-semiconductor-metal traveling-wave photodetector (MSM-TWPD) due to its high power-bandwidth product [10] and coplanar-waveguide-fed slot antennas due to its easy connection with planar devices and higher radiation power than the spiral antennas at the resonant frequency. The detailed structure and design of TWPD were described in [11]. The absorption length of the TWPD employed in the photonic transmitter is $50 \mu \mathrm{m}$ while the center strip and air gap widths are 2 and $0.3 \mu \mathrm{m}$, respectively. The RF choke filter, which acts as an inductance [12], avoids the high frequency ac current (with a resonant frequency of the slot antenna) leaking into the dc probe pad that will lower the radiation efficiency. Please note that this new structure does not need a quarter-wavelength impedance transformer between the MSM-TWPD and the slot antenna compared with the previous structure [5] because the impedance of the slot antenna had been designed to match that of the MSM-TWPD (50 $\Omega$ ). Therefore, the microwave propagation loss in the impedance transformer can be avoided and more power can be radiated from the antenna. 


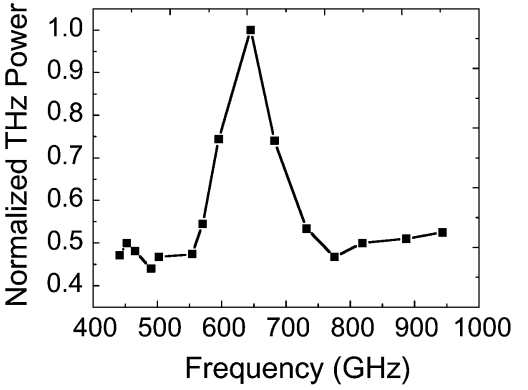

Fig. 2. Measured frequency response of the photonic transmitter with an antenna resonating at $\sim 650 \mathrm{GHz}$.

All devices reported in this study were fabricated on a LTG-GaAs layer on top of an AlGaAs cladding layer with antenna resonating at $\sim 650 \mathrm{GHz}$. The GaAs substrate was removed to form a membrane structure whose total thickness is $\sim 5 \mu \mathrm{m}$. The membrane device was then mounted on a glass substrate [5]. To study the device physics and saturation behaviors, we excited the photonic transmitters by using broad optical pulses with temporal modulations inside the pulse envelopes with a central wavelength of $780 \mathrm{~nm}$. The broad optical modulated-pulses were created by passing a femtosecond pulse train, with an 82-MHz repetition rate, through a tunable high finesse Fabry-Pérot filter and the modulation frequency is determined by filter's free spectral range (FSR) [13]. The optical beam was focused into the absorption region of the device by an objective. With such an excitation, photonic transmitters can generate quasi-continuous-wave $(\mathrm{CW})$ narrow-band $\mathrm{THz}$ radiation corresponding to the filter's FSR frequency [14], [15]. The excited $\mathrm{THz}$ waves radiated from the glass substrate side into the free space were collected and focused onto the detection window of a bolometer, which has a Winston cone inside, by two off-axis paraboloidal mirrors. By using a liquid-helium-cooled $\mathrm{Si}$ bolometer calibrated with a blackbody radiation source [5], the average radiation power emitted from the photonic transmitter can be measured. The $\mathrm{THz}$ beam propagation loss in air was also measured and calibrated by measuring the $\mathrm{THz}$ power as a function of propagation distance. The obtained propagation loss at $645 \mathrm{GHz}$ was about $0.069 \mathrm{~cm}^{-1}$. By tuning the spacing of the Fabry-Pérot filter, the modulation frequency of the optical excitation onto the photonic transmitter can be modified so that we can measure the frequency response of the photonic transmitter. The measured frequency response of the photonic transmitter is shown in Fig. 2. The measured resonant frequency of the photonic transmitter is $645 \mathrm{GHz}$, which is close to our designed antenna resonant frequency (680 $\mathrm{GHz}$ ), indicating the dominant role of antenna on the radiating efficiency.

For the device (shown in Fig. 1) operating at its antenna's resonant frequency of $645 \mathrm{GHz}$, by fixing the FSR of the optical filter at this specific frequency, the measured external conversion efficiency (including the coupling loss into the TWPD, the loss in the glass substrate, and loss in the collection system) versus applied bias voltages under $0.93-\mathrm{mW}$ average-power optical excitation is shown in Fig. 3. The conversion efficiency of the device increases with increased bias and starts to show some saturation behavior if the bias is higher than $8 \mathrm{~V}$ [5]. Serious de-

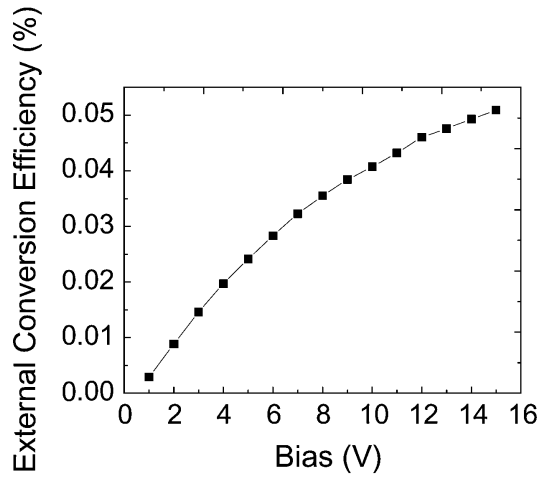

Fig. 3. External conversion efficiency of the photonic transmitter versus detector bias under $0.93-\mathrm{mW}$ optical excitation.

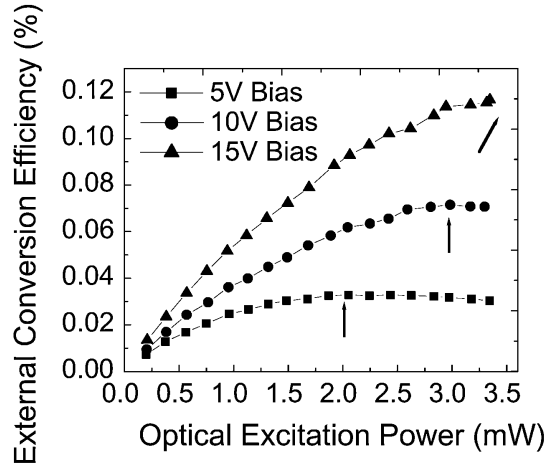

Fig. 4. Optical excitation power-dependent external conversion efficiency under different detector biases.

tector bandwidth degradation is expected when the bias is higher than $20 \mathrm{~V}$ due to the Coulomb barrier lowering effect [10], [16]. However, our current device could not sustain bias higher than $15 \mathrm{~V}$ because the gap between the electrodes is so narrow $(0.3 \mu \mathrm{m})$ that high bias voltages will generate high electrical field near the gap causing device breakdown. According to previous studies on MSM-TWPD [10], the observed sublinear behavior above $8 \mathrm{~V}$ is attributed to carrier lifetime increasing effect in LTG-GaAs [5]. Our recent study [8] reveals the fact that when the applied bias on the MSM-TWPD is higher than $6 \mathrm{~V}$, high field-induced intervalley scatterings and, thus the resulting hot electron effect, will slow down the electron relaxation time as well as its capture rate into the defect states, thus lengthening the electron lifetime and degrading the bandwidth of MSM-TWPD. With a narrower photodetector bandwidth, lower than $645 \mathrm{GHz}$, the conversion efficiency of the photonic transmitter, thus, starts to show the trend of saturation at bias higher than $8 \mathrm{~V}$. However, higher conversion efficiency is still obtained with higher bias, at least up to $15 \mathrm{~V}$, due to improved current response.

Beside bias, another important factor to influence the conversion efficiency, will be the optical excitation energy and thus the generated photocarrier density. By fixing the bias at $5 \mathrm{~V}$, we studied the excitation power-dependent external conversion efficiency (squares in Fig. 4). Under 5-V bias, the external conversion efficiency saturates if the average optical excitation power is higher than $2 \mathrm{~mW}$, corresponding to a pulse energy of $24 \mathrm{pJ}$. According to our previous study of the MSM-TWPDs, this conversion efficiency saturation phenomenon under high excitation power should be attributed to the high photocarrier density re- 
sulted defect saturation behavior in the LTG-GaAs active layer [9] so that the carrier lifetime will increase, causing the bandwidth of the MSM-TWPD to degrade. On the other hand, these high-density photocarriers screen the applied bias field so that the induced current does not increase much as the excitation power increased, resulting in lower conversion efficiency.

In order to consider the influences of detector bias and optical excitation power to the photonic transmitter saturation behavior at the same time, we also studied the excitation powerdependent conversion efficiency under different detector bias voltages (Fig. 4). It is interesting to see that when the detector bias increased, the saturation occurred at higher optical excitation power (arrows in Fig. 4). This is because space charge screening effect induced by the high carrier density [17], [18], that strongly affects the photocurrent response, can be released by applying higher electrical field, thus recovering the photocurrent response. It is, thus, important to find that the optimized conversion efficiency occurs with both high optical excitation and high detector bias, even though these two effects individually would strongly degrade the detector bandwidth and transmitter efficiency behaviors. Limited by the maximum available optical average power of $3.5 \mathrm{~mW}$ and maximum tolerable applied bias of $15 \mathrm{~V}$, the optimal external conversion efficiency of our new device with $645-\mathrm{GHz}$ radiation is $0.11 \%$ (corresponding to $3.9-\mu \mathrm{W}$ average power and $13.2-\mathrm{mW}$ peak power after considering loss in air). We did not calibrate the loss in the coupling and collection system. The signal-to-noise ratio of the system is higher than $80 \mathrm{~dB}$. According to our knowledge, this value is the highest reported external conversion efficiency of all photonic transmitters with radiation higher than $500 \mathrm{GHz}$ and is about 15 times higher than the optimum value that has been reported [19] at a similar radiation frequency.

In summary, the saturation behaviors of the edge-coupled membrane photonic transmitters were studied under 780-nm quasi-CW optical excitations. The saturation behaviors determine the optimum device operation condition, which was influenced by detector bias and optical excitation power at the same time. By considering these two factors together, we have successfully uncovered the optimum operation condition for photonic transmitter and a maximum external light- $\mathrm{THz}$ conversion efficiency of $0.11 \%$ was achieved with $645-\mathrm{GHz}$ radiation. The ultrahigh conversion efficiency of our device is based on two factors: First, a high-speed and high-power MSM-TWPD is employed and second, a membrane structure of the photonic transmitter is utilized. With such a high conversion efficiency of the edge-coupled membrane structures, a compact $\mathrm{THz}$ source integrated with semiconductor lasers is possible to be realized.

\section{ACKNOWLEDGMENT}

The authors would like to acknowledge the technical support of J.-W. Shi, H.-L. Liu, and Shey-Shi Lu.

\section{REFERENCES}

[1] H. Eisele, A. Rydberg, and G. I. Haddad, "Recent advances in the performance of InP Gunn devices and GaAs TUNNET diodes for the 100-300-GHz frequency range and above," IEEE Trans. Microwave Theory Tech., vol. 48, pp. 626-631, Apr. 2000.

[2] Y. P. Gousev, I. V. Altukhov, K. A. Korolev, V. P. Sinis, M. S. Kagan, E. E. Haller, M. A. Odnoblyudov, I. N. Yassievich, and K.-A. Chao, "Widely tunable continuous-wave THz laser," Appl. Phys. Lett., vol. 75, pp. 757-759, 1999.

[3] S. Barbieri, J. Alton, S. S. Dhillon, H. E. Beere, M. Evans, E. H. Linfield, A. G. Davies, D. A. Ritchie, R. Kohler, A. Tredicucci, and F. Beltram, "Continuous-wave operation of terahertz quantum-cascade lasers,” IEEE J. Quantum Electron., vol. 39, pp. 586-591, Apr. 2003.

[4] M. Reddy, S. C. Martin, A. C. Molnar, R. E. Muller, R. P. Smith, P. H. Siegel, M. J. Mondry, M. J. W. Rodwell, H. Kroemer, and S. J. Allen Jr., "Monolithic Schottky-collector resonant tunnel diode oscillator arrays to $650 \mathrm{GHz}$," IEEE Electron Device Lett., vol. 18, pp. 218-221, May 1997.

[5] J.-W. Shi, S.-W. Chu, M.-C. Tien, C.-K. Sun, Y.-J. Chiu, and J. E. Bowers, "Edge-coupled membrane terahertz photonic transmitters based on metal-semiconductor-metal traveling-wave photodetectors," Appl. Phys. Lett., vol. 81, pp. 5108-5110, 2002.

[6] K. J. Siebert, H. Quast, R. Leonhardt, T. Loffler, M. Thomson, and S. Czasch, "Continuous-wave all-optoelectronic terahertz imaging," Appl. Phys. Lett., vol. 80, pp. 3003-3005, 2002.

[7] P. Chen, G. A. Blake, M. C. Gaidis, E. R. Brown, K. A. McIntosh, S. Y. Chou, M. I. Nathan, and F. Williamson, "Spectroscopic applications and frequency locking of THz photomixing with distributed-Bragg-reflector diode lasers in low-temperature-grown GaAs," Appl. Phys. Lett., vol. 71, pp. 1601-1603, 1997.

[8] C.-K. Sun, Y.-H. Chen, J.-W. Shi, Y.-J. Chiu, K.-G. Gan, and J. E. Bowers, "Electron relaxation and transport dynamics in low-temperature-grown GaAs under $1 \mathrm{eV}$ optical excitation," Appl. Phys. Lett., vol. 83, pp. 911-913, 2003.

[9] K.-G. Gan, J.-W. Shi, Y.-H. Chen, C.-K. Sun, Y.-J. Chiu, and J. E. Bowers, "Ultrahigh power-bandwidth-product performance of low-temperature-grown GaAs based metal-semiconductor-metal traveling-wave photodetectors," Appl. Phys. Lett., vol. 80, pp. 4054-4056, 2002.

[10] J.-W. Shi, K.-G. Gan, Y.-H. Chen, C.-K. Sun, Y.-J. Chiu, and J. E. Bowers, "Ultrahigh-power-bandwidth product and nonlinear photoconductance performances of low-temperature-grown GaAs-based metal-semiconductor-metal traveling-wave photodetectors," IEEE Photon. Technol. Lett., vol. 14, pp. 1587-1589, Nov. 2002.

[11] J.-W. Shi, K.-G. Gan, Y.-J. Chiu, Y.-H. Chen, C.-K. Sun, Y.-J. Yang, and J. E. Bowers, "Metal-semiconductor-metal traveling-wave photodetectors," IEEE Photon. Technol. Lett., vol. 13, pp. 623-625, June 2001.

[12] M. Tani, K.-S. Lee, and X.-C. Zhang, "Detection of terahertz radiation with low-temperature-grown GaAs-based photoconductive antenna using $1.55 \mu \mathrm{m}$ probe," Appl. Phys. Lett., vol. 77, pp. 1396-1398, 2000.

[13] D. A. Yanson, M. W. Street, S. D. McDougall, I. G. Thayne, J. H. Marsh, and E. A. Avrutin, "Ultrafast harmonic mode-locking of monolithic compound-cavity laser diodes incorporating photonic-bandgap reflectors," IEEE J. Quantum Electron., vol. 38, pp. 1-11, Jan. 2002.

[14] A. S. Weling, B. B. Hu, N. M. Froberg, and D. H. Auston, "Generation of tunable narrow-band $\mathrm{THz}$ radiation from large aperture photoconducting antennas," Appl. Phys. Lett., vol. 64, pp. 137-139, 1994.

[15] Y. Liu, S.-G. Park, and A. M. Weiner, "Enhancement of narrow-band terahertz radiation from photoconducting antennas by optical pulse shaping," Opt. Lett., vol. 21, pp. 1762-1764, 1996.

[16] N. Zamdmer, Q. Hu, K. A. Mclntosh, and S. Verghese, "Increase in response time of low-temperature-grown GaAs photoconductive switches at high voltage bias," Appl. Phys. Lett., vol. 75, pp. 2313-2314, 1999.

[17] C.-K. Sun, I.-H. Tan, and J. E. Bowers, "Ultrafast transport dynamics of p-i-n photodetectors under high power illumination," IEEE Photon. Technol. Lett., vol. 10, pp. 135-137, Jan. 1998.

[18] Y.-L. Huang and C.-K. Sun, "Nonlinear saturation behaviors of high-speed p-i-n photodetectors," J. Lightwave Technol., vol. 18, pp. 203-212, Feb. 2000.

[19] S. M. Duffy, S. Verghese, K. A. McIntosh, A. Jackson, A. C. Gossard, and S. Matsuura, "Accurate modeling of dual dipole and slot elements used with photomixers for coherent terahertz output power," IEEE Trans. Microwave Theory Tech., vol. 49, pp. 1032-1038, June 2001. 